\title{
A remark on the Laplacian flow and the modified Laplacian co-flow in $\mathrm{G}_{2}$-geometry
}

\author{
Lucio Bedulli $^{1} \cdot$ Luigi Vezzoni $^{2}$ (D)
}

Received: 15 May 2020 / Accepted: 7 July 2020 / Published online: 23 July 2020

(c) The Author(s) 2020

\begin{abstract}
We give a shorter proof of the well-posedness of the Laplacian flow in $\mathrm{G}_{2}$-geometry. This is based on the observation that the DeTurck-Laplacian flow of $\mathrm{G}_{2}$-structures introduced by Bryant and $\mathrm{Xu}$ as a gauge fixing of the Laplacian flow can be regarded as a flow of (not necessarily closed) $\mathrm{G}_{2}$-structures, which fits in the general framework introduced by Hamilton in J Differ Geom 17(2):255-306, 1982. A similar application is given for the modified Laplacian co-flow.
\end{abstract}

Keywords Laplacian flow · G2-geometry $\cdot$ Short-time existence

\section{Introduction}

$\operatorname{In}[1]$ Bryant introduced a geometric flow in $\mathrm{G}_{2}$-geometry which evolves an initial closed $\mathrm{G}_{2}$-structure $\varphi_{0}$ in the direction of its Laplacian.

Given a compact seven-dimensional manifold with a closed $\mathrm{G}_{2}$-structure $\left(M, \varphi_{0}\right)$, a Laplacian flow is a solution to the evolution equation

$$
\frac{\partial}{\partial t} \varphi_{t}=\Delta_{\varphi_{t}} \varphi_{t}, \quad d \varphi_{t}=0, \quad \varphi_{\mid t=0}=\varphi_{0} .
$$

The well-posedness of Eq. (1) is proved in[2] by applying the Nash-Moser theorem to the gauge fixing

$$
\frac{\partial}{\partial t} \varphi_{t}=\Delta_{\varphi_{t}} \varphi_{t}+\mathcal{L}_{V\left(\varphi_{t}\right)} \varphi_{t}, \quad d \varphi_{t}=0, \quad \varphi_{\mid t=0}=\varphi_{0},
$$

where $\mathcal{L}$ is the Lie derivative and $V: C^{\infty}\left(M, \Lambda_{+}^{3}\right) \rightarrow C^{\infty}(M, T M)$ is a first-order differential operator which depends on the choice of a connection on $M$. Here, $\Lambda_{+}^{3}$ denotes the open

Luigi Vezzoni

luigi.vezzoni@unito.it

Lucio Bedulli

lucio.bedulli@univaq.it

1 Dipartimento di Ingegneria e Scienze dell'Informazione e Matematica, Università dell'Aquila, Via Vetoio 67100, L'Aquila, Italy

2 Dipartimento di Matematica G. Peano, Università di Torino, Via Carlo Alberto 10, 10123 Turin, Italy 
subbundle of $\Lambda^{3}$ of $\mathrm{G}_{2}$-structures on $M$. A solution to (2) is usually called a DeTurck-Laplacian flow.

A DeTurck-Laplacian flow $\varphi_{t}$ is also a solution to

$$
\frac{\partial}{\partial t} \varphi_{t}=d d_{\varphi_{t}}^{*} \varphi_{t}+d l_{V\left(\varphi_{t}\right)} \varphi_{t}, \quad \varphi_{\mid t=0}=\varphi_{0} .
$$

In the present note, we observe that Eq. (3) fits in the general framework introduced by Hamilton in[4]. As a direct consequence, we have the following theorem which in particular implies the well-posedness of (2)

Theorem 1.1 Let $\left(M, \varphi_{0}\right)$ be a compact seven-dimensional manifold with a $\mathrm{G}_{2}$-structure. Then, Eq. (3) has a unique short-time solution.

In[5] Karigiannis, McKay and Tsui introduced the Laplacian co-flow as the solution to the evolution equation

$$
\frac{\partial}{\partial t}\left(*_{\varphi_{t}} \varphi_{t}\right)=-\Delta_{\varphi_{t}} *_{\varphi_{t}} \varphi_{t}, \quad d *_{\varphi_{t}} \varphi_{t}=0, \quad \varphi_{\mid t=0}=\varphi_{0},
$$

where in this case $\varphi_{0}$ is supposed to be co-closed with respect to the metric induced by itself. The well-posedness of this last equation is still an open problem and Grigorian introduced in[3] the following modification

$$
\frac{\partial}{\partial t}\left(*_{\varphi_{t}} \varphi_{t}\right)=\Delta_{\varphi_{t}} * \varphi_{t} \varphi_{t}+2 d\left(\left(A-\operatorname{Tr}\left(T\left(\varphi_{t}\right)\right) \varphi_{t}\right), \quad d * \varphi_{t} \varphi_{t}=0, \quad \varphi_{\mid t=0}=\varphi_{0},\right.
$$

where $A$ is a constant and $T\left(\varphi_{t}\right)$ is the torsion of $\varphi_{t}$. $\operatorname{In}[3]$, the well-posedness of (5) is proved following the same approach of Bryant in[1] by applying the Nash-Moser theorem to the gauge fixing

$$
\frac{\partial}{\partial t}\left(*_{\varphi_{t}} \varphi_{t}\right)=\Delta_{\varphi_{t}} *_{\varphi_{t}} \varphi_{t}+2 d\left(\left(A-\operatorname{Tr}\left(T\left(\varphi_{t}\right)\right) \varphi_{t}\right)+\mathcal{L}_{V\left(\varphi_{t}\right)} \varphi_{t}, \quad d *_{\varphi_{t}} \varphi_{t}=0, \quad \varphi_{\mid t=0}=\varphi_{0}\right.
$$

Any solution to Eq. (6) satisfies

$$
\frac{\partial}{\partial t}\left(*_{\varphi_{t}} \varphi_{t}\right)=d d_{\varphi_{t}}^{*} *_{\varphi_{t}} \varphi_{t}+2 d\left(\left(A-\operatorname{Tr}\left(T\left(\varphi_{t}\right)\right) \varphi_{t}\right)+d l_{V\left(\varphi_{t}\right)} \varphi_{t}, \quad \varphi_{\mid t=0}=\varphi_{0},\right.
$$

Analogously to Theorem 1.1, we have

Theorem 1.2 Let $\left(M, \varphi_{0}\right)$ be a compact seven-dimensional manifold with a $\mathrm{G}_{2}$-structure. Then, Eq. (6) has a unique short-time solution.

\section{Proof of the results}

Both Theorems 1.1 and 1.2 can be proved by using the following setup introduced by Hamilton in [4].

Let $M$ be an oriented compact manifold, $F$ a vector bundle over $M, U$ an open subbundle of $F$ and

$$
E: C^{\infty}(M, U) \rightarrow C^{\infty}(M, F)
$$


a second-order differential operator. For $f \in C^{\infty}(M, U)$, we denote by $D E(f): C^{\infty}(M, F) \rightarrow C^{\infty}(M, F)$ the linearization of $E$ at $f$ and by $\sigma D E(f)$ the principal symbol of $D E(f)$.

Definition 2.1 An integrability condition for $E$ is a first-order linear differential operator

$$
L: C^{\infty}(M, F) \rightarrow C^{\infty}(M, G),
$$

where $G$ is another vector bundle over $M$, such that $L(E(f))=0$ for all $f \in C^{\infty}(M, U)$, and for every $(x, \xi)$ in $T^{*} M$ all the eigenvalues of $\sigma D E(f)(x, \xi)$ restricted to $\operatorname{ker} \sigma L(x, \xi)$ have strictly positive real part.

Theorem 2.1 (Hamilton [4, Theorem 5.1]) Assume that E admits an integrability condition. Then, for every $f_{0} \in C^{\infty}(M, U)$ the geometric flow

$$
\frac{\partial f}{\partial t}=E(f), \quad f(0)=f_{0},
$$

has a unique short-time solution.

Now we can focus on the setup of Theorem 1.1. Here, we consider

$$
F=\Lambda^{3}, \quad U=\Lambda_{+}^{3}, \quad G=\Lambda^{4}, \quad E(\varphi)=d d_{\varphi}^{*} \varphi+d l_{V(\varphi)} \varphi, \quad L=d: C^{\infty}\left(M, \Lambda^{3}\right) \rightarrow C^{\infty}\left(M, \Lambda^{4}\right) .
$$

From [2], it follows that for every $\varphi \in C^{\infty}(M, U)$ and every closed $\psi \in C^{\infty}\left(M, \Lambda^{3}\right)$, we have

$$
D E(\varphi)(\psi)=-\Delta_{\varphi} \psi+\text { l.o.t. }
$$

Hence, all the assumptions of Hamilton's Theorem 2.1 are satisfied and Theorem 1.1 follows.

Notice that if the starting form $\varphi_{0}$ is closed, then the solution to (3) is closed for every $t$ since

$$
d \frac{\partial}{\partial t} \varphi=0 .
$$

Therefore, if $\varphi_{0}$ is closed, the unique solution $\varphi_{t}$ to (3) solves also the DeTurck-Laplacian flow (2) and the short-time existence of the DeTurck-Laplacian flow (2) can be deduced from Theorem 1.1.

About the proof of Theorem 1.2, we set

$$
\begin{aligned}
& F=\Lambda^{4}, \quad U=\Lambda_{+}^{4}, \quad G=\Lambda^{5}, \quad E\left(*_{\varphi} \varphi\right)=d d_{\varphi}^{*} \varphi+d l_{V(\varphi)}+2 d((A-\operatorname{Tr}(T(\varphi)) \varphi), \\
& L=d: C^{\infty}\left(M, \Lambda^{4}\right) \rightarrow C^{\infty}\left(M, \Lambda^{5}\right) .
\end{aligned}
$$

From [3], it follows

$$
D E\left(*_{\varphi} \varphi\right)(\psi)=-\Delta_{\varphi} \psi+\text { l.o.t. }
$$

for every closed $\psi \in C^{\infty}\left(M, \Lambda^{4}\right)$ and the proof of Theorem 1.2 follows.

Acknowledgements Open access funding provided by Università degli Studi di Torino within the CRUICARE Agreement. We would like to thank Jason Lotay for useful conversations. 
Funding This work was supported by GNSAGA of INdAM.

Open Access This article is licensed under a Creative Commons Attribution 4.0 International License, which permits use, sharing, adaptation, distribution and reproduction in any medium or format, as long as you give appropriate credit to the original author(s) and the source, provide a link to the Creative Commons licence, and indicate if changes were made. The images or other third party material in this article are included in the article's Creative Commons licence, unless indicated otherwise in a credit line to the material. If material is not included in the article's Creative Commons licence and your intended use is not permitted by statutory regulation or exceeds the permitted use, you will need to obtain permission directly from the copyright holder. To view a copy of this licence, visit http://creativecommons.org/licenses/by/4.0/.

\section{References}

1. Bryant, R.: Some remarks on $\mathrm{G}_{2}$-structures. In: Proceedings of Gokova Geometry/Topology Conference, Gokova, pp. 75-109 (2006)

2. Bryant, R., Xu, F.: Laplacian Flow for Closed $\mathrm{G}_{2}$-Structures: Short Time Behavior. arXiv:1101.2004

3. Grigorian, S.: Short-time behaviour of a modified Laplacian coflow of $\mathrm{G}_{2}$-structures. Adv. Math. 248, 378-415 (2013)

4. Hamilton, R.S.: Three-manifolds with positive Ricci curvature. J. Differential Geom. 17(2), 255-306 (1982)

5. Karigiannis, S., McKay, B., Tsui, M.-P.: Soliton solutions for the Laplacian coflow of some $\mathrm{G}_{2}$ structures with symmetry. Differential Geom. Appl. 30, 318-333 (2012)

Publisher's Note Springer Nature remains neutral with regard to jurisdictional claims in published maps and institutional affiliations. 